\title{
Analysis on the Clinical Effectiveness of Perioperative Period Nursing Care of Infants
}

\author{
Suxiang Yue* \\ No. 4 People's Hospital of Linfen, Linfen, Shanxi 041000, China
}

\begin{abstract}
Objective: To explore the physiological and psychological nursing and nursing methods for infants during operation. Methods: The nursing measures are taken before, during and after operation for 138 infants that have received surgical treatment in our hospital from February 2013 to January 2015 after close observation. Results: The operations for all 138 infants have been completed successfully without any surgical complications. Conclusion: The sufficient pre-operative nursing for infants, the comfortable body position, suitable heat preservation and close observation during operation and the recovery from general anesthesia after operation are crucial to peri-operative nursing for infants.
\end{abstract}

\author{
KEYWORDS \\ Infants \\ Perioperative period \\ Clinical effectiveness
}

\section{Introduction}

Infants have relatively poor physiological function and storage capacity and their tissues and organs are not developed maturely. Also, infants are very young and light, and have much physiological and anatomical difference from grownups. Infants are more vulnerable to external interference and diseases, and cannot receive the same dose of drugs as grownups. Hence, they are less capable to withstand operations [1]. Along with the gradual development of surgical treatment for infants, the supporting nursing pattern has been gradually improved and reformed. Our hospital provides the all-inclusive nursing for 138 infants that receive operations from February 2013 to January 2015 through good pre-operative preparation, specific management during operation and active post-operative treatment, etc., and achieves great effect in operations. Now, several issues that should be kept in mind during the surgical process for infants are summarized in the following sections.

\section{Data and methods}

\subsection{General data}

Our hospital provides operations for 138 infants from Feb-

Copyright $\odot 2015$ Suxiang Yue

doi: $10.18686 /$ jn.v4i1.5

Received: January 18, 2015; Accepted: February 26, 2015; Published online: March 28, 2015

This is an open-access article distributed under the terms of the Creative Commons Attribution Unported License (http://creativecommons.org/ licenses/by-nc/4.0/), which permits unrestricted use, distribution, and reproduction in any medium, provided the original work is properly cited.

${ }^{\star}$ Corresponding author: No. 4 People's Hospital of Linfen, Linfen, Shanxi 041000, China. E-mail: yue_sx407@126.com ruary 2013 to January 2015. Among them, there are 27 infants of 1 to 12 months old and 111 infants of 1 to 3 years old. These infants receive operations with 76 cases for indirect inguinal hernia, 19 cases for incarcerated indirect hernia, 5 cases for undescended testis, 24 cases for funicular hydrocele, 3 cases for intussusception and other cases for lymphoma and traumatic fracture, etc. After operation, all 138 infants are cured and discharged without any surgical complications.

\subsection{Methods}

\subsubsection{Pre-operative nursing}

(1) Pre-operative preparation. Before operation, nurses should learn carefully about the condition of an infant that is sick. The nurses in operating room should be familiar with the psychological features of the infant to reduce the psychological trauma of the infant to the maximum. Nurses should carefully explain the precautions of operation to the infant's family, and give friendly and reasonable psychological comfort based on the age of the infant and the corresponding psychological reaction of the infant, in order to alleviate the fear of the infant. Moreover, nurses should make the infant's family know some about the process of operation, and introduce the equipment and environment of operating room to them, and should hint that the surgeon and anesthetist responsible for the infant's operation are highly skilled, in order to make the family feel confident and eliminate their doubt about the operation. The relationships between doctor and patient and between nurse and patient should be further improved [2]. As an infant has strong sense of dependence on his mother, the 
separation from mother may cause some psychological trauma to him. Hence, the time of operation should be reduced as much as possible. Shortening the time of separating infant from other is a basic principle followed to protect the psychological condition of infant during operation [3]. After being taken away from mother, an infant may easily cry, which causes hypoxia, flatulence and cyanosis. In order to shorten the separation of mother from infant, the parents may keep company with the infant before operation for general anesthesia. After the infant sleeps deeply, he can be taken into operating room.

(2) Pre-operative visit: After the date of operation is determined, patrol nurse pays a visit to the infant and his family in the ward one day before the date, learns about the general condition of the infant, e.g. weight, peripheral vascular and skin condition, and explains the purpose and significance of stopping eating and drinking in simple words based on the age of infant and the educational background of parents. Before operation, the infant should stop eating for 4-6 hours and stop drinking for 2-3 hours, in order to guarantee empty stomach, which can prevent backflow, vomiting or aspiration by mistake of stomach contents during operation. Through body language, e.g. gentle touch, hug and teasing, the nurse can have contact with the infant to reduce his fear. Moreover, the nurse should answer the questions asked by the parents patiently to alleviate their concern about the operation. After receiving the notice of operation for any emergency treatment, if the condition of the infant permits, the patrol nurse should pay a personal visit to the infant and his family to learn about the condition of the infant and give some instructions, in order to alleviate their worry and concern [4].

\subsubsection{Nursing during operation}

(1) Body position: The infant should be placed according to the requirements of operation. For his tender skin, the infant should be placed on the flat and soft pad, and covered by a dry fabric without wrinkles. When the infant lies on his back, soft cushion should be placed under his shoulders, and his head should be lifted backward and faced one side. The upper limbs should be extended by no more than $90^{\circ}$. When the infant lies on his side, soft cushion should be placed under the elbows and between the legs. The upper limb on the upper side should be wrapped by cotton pad, and suspended on the anesthetic frame at the functional position, while the lower limb on the upper side is naturally bent and the lower limb on the lower side is straightened. When the infant lies on his chest, hollow soft cushion should be placed under the chest and abdomen to the gap enough to insert two fingers horizontally, and soft cushion should be placed at knees to make them bend by $10 \sim 15^{\circ}$. The toes are suspended and the angles are bent backward. When the infant is placed at lithotomy position, the support should be as tall as the thighs when the buttocks bend at supine position, and soft cushion should be placed under the buttocks. The lower limbs should be separated by no more than $90^{\circ}[5]$.

(2) Venous passage. The common positions include scalp vein, external carotid, dorsal vein of foot, radial vein or great saphenous vein. It uses $22-24 \mathrm{G}$ venous keeping needle with wing. The position should be selected according to the needs of operation. Among them, radial vein or great saphenous vein are often utilized, and also guarantee the highest success rate of one-time puncture (higher than 90\%). After venous passage is created, aseptic cotton ball is pressed at the puncture, and fixed with a transparent plastic film [6].

(3) Nasogastric tube and urinal catheter indwelling: When gastric tube is placed through nostril, Ch $8 \sim 10$ urinary catheter should be used to replace gastric tube. When it is inserted into through nostril to the length from the wing of nose to earlobe, it is placed under the guidance of telescope [7]. When gastric tube is inserted through mouth, it ins inserted slowly through the infant's bite block passage. As an infant has narrow urethra and tender skin, urinal catheter should be inserted gently to prevent it from damaging the infant's mucous membrane of urethra, especially boys. Normally, the tiny tube at the front end of infusion is used by cutting off the needle and made into slight arc. Then, urinal catheter is indwelled based on the ordinary catheterization process. When urinal output is $>20$ $\mathrm{mL} / \mathrm{h}$ for child or $>10 \mathrm{~mL} / \mathrm{h}$ for baby, the infant may have normal kidney function [8].

(4) Maintenance of body temperature: During operation, the body temperature of infant is easily affected by various physical and chemical factors. Normally, the body temperature of a baby less than 1 year old may easily drop, while the body temperature of a child over 1 year old may easily go up. When an infant has low body temperature or a fever, it may easily develop some severe complications, even causing the irreversible damages to brain tissues and liver cells. Therefore, attention should be paid to the regular monitoring of body temperature during operation, and maintaining the indoor temperature of operating room is the easiest and most effective method to keep the proper body temperature of infant. Generally, the indoor temperature should be kept around $25{ }^{\circ} \mathrm{C}$ with the relative humidity of around $65 \%$. If an infant has low body temperature, it should be increased by using hot water bottle or placing electric blanket, etc. If an infant has a fever, the body temperature can be lowered through physical cooling.

(5) Skin protection: An infant has tender skin, so disinfection should use less irritating disinfectant, normally including povidone $(0.5 \%)$ and Benzalkonium Bromide. The dressing on cut should use as less tape as possible, but be wrapped using pad and bandage, or using abdominal binder. The wrapping should not be too tight, as it may affect the breath of infant.

\subsubsection{Consciousness recovering period}

(1) Keep the smoothness of respiratory tract: If an infant is 
still unconscious, aspirator and mucous extractor should be prepared to assist the anesthetist to suction the sputum if any, and help the anesthetist raise the lower jaw of the infant and insert the oro-pharyngeal tube if necessary, in order to achieve the smoothness of respiratory tract. Close attention should be paid to observing the infant's smoothness of respiratory tract, amplitude of respiration and rhythm of respiration. (2) Guarantee the stability of circulation: Monitor the change of circulation system. For instance, it is necessary to observe the infant's heart rate, oxygen saturation of finger vein, skin color and urinal output, etc. (3) Observe body temperature: After operation, attention should be paid to the change of the infant's body temperature. In summer, attention should be paid to preventing fever. In winter, the infant should be kept warm in order to prevent it from affecting the recovery of consciousness. (4) Before recovering consciousness, an infant often experiences agitation, so attention should be paid to protection against any accident. (5) When an infant recovers consciousness completely and the vital signs are stable, the ward is notified to prepare oxygen and sputum suction devices. Then, the infant can be escorted by anesthetist back to the ward, and the condition of the infant and the precautions should be told to the ward nurse face to face [9].

\subsubsection{Post-operative nursing}

(1) Oxygen inhalation: Regular low-concentration continuous oxygen inhalation is provided at the flow rate of 2-4 L/min. When unrepeated device is used, the inhaled oxygen should be warmed and humidified. Clear mask is used in oxygen inhalation to prevent accumulation of $\mathrm{CO}_{2}$. Also, attention should be paid to the change of respiration through lip color, identifying and eliminating the secretions of respiratory tract in a timely manner.

(2) Aspiration of sputum: The sputum aspirator tube should be thin and flexible, and its diameter is $1 / 2$ of the inner diameter of tracheal tube. During aspiration, the pressure should not be higher than $200 \mathrm{mmHg}$ for no more than $10 \mathrm{~s}$. The aspiration should be performed orally as much as possible. The aspiration of sputum should not be carried out during insertion. During removal of the tube, the tube should be turned while removing and aspirating, and should not be stopped at any position.

\subsubsection{Return visit after operation}

The nursing for surgery of infants should be constantly explored. We keep visiting the ward and asking about the healing of the infant's wound, the pain of the infant and the opinions of their parents on the $3^{\text {rd }}$ day and the $7^{\text {th }}$ day after operation, in order to constantly improve the work and their satisfaction with nursing.

\section{Discussion}

The operation of infants is different from that of grownups. As it needs small and delicate apparatuses and items, op- erating room should be equipped with special articles and apparatuses for operation of infants, in order to facilitate anesthesia and operation. Infants are psychologically immature and very vulnerable, so nurses in operating room should be more careful, patient and warm-hearted in the nursing of infant, and show stronger motherly love and sense of responsibility, in order to make the infant and parents feel comfortable, warm and safe.

With regard to anatomical and physiological characteristics and categories of diseases, the operation of infants is much different from that of grownups, so the surgical theories for adults should not be borrowed mechanically in the operation of infants. During the surgical process of infants, nurses in operating room should strictly implement the procedures for operation in an accurate, dexterous and delicate manner. The fundamental nursing measures should be constantly improved, especially body position of infant and venous puncture, etc., in order to shorten the waiting time of parents. We should pay close attention to the condition of the patient and the progress of the operation, keep calm during any emergency, waste no time and make all efforts to avoid any accident during operation. In this group of cases, there is no any problem of nursing that threatens the life of the infants or obstructs the successful implementation of operation. Above all, nurses in operating room should master common surgical skills, and have the knowledge of pediatrics and the psychology of infants, in order to better assist the surgeon and ensure the safe and successful operation of infants.

\section{References}

1. Zhu D, Zhou L. Key points in peri-operative nursing for infants. Nursing in operating room. Beijing: People's Medical Publishing House. 2008;1:173.

2. Liu J, Zhao J. Modern Anesthesiology. Monitoring and management during anesthesia. Beijing: People's Medical Publishing House. 1997:84.

3. Jiang $X$, Sun $Y$, Guan B. Study on the safety management of operating patients. China Medical Herald. 2007;4(16):161-162.

4. Cao X. Cleanliness management of laminar flow operating room. Chinese Journal of Nosocomiology. 2009;19(11):1394.

5. Pei H. Experience in overall nursing for operating patients. Jilin Medical Journal. 2011;32(26):5610.

6. Zeng F, Feng $S$, He G. Exploration in the cooperation of nursing for infant operation. The Medical Forum. 2012; 16(18):2423.

7. Jiang $X$, Sun $Y$, Guan B. Study on the safety management of operating patients. China Medical Herald. 2007;4(16):161-162.

8. Cao X. Cleanliness management of laminar flow operating room. Chinese Journal of Nosocomiology. 2009;19(11):1394.

9. Pei $\mathrm{H}$. Experience in overall nursing for operating patients. Jilin Medical Journal, 2011;32(26):5610. 